\title{
Effects of exogenous melatonin on antioxidant activity of kiwi seedlings
}

\section{under weak light}

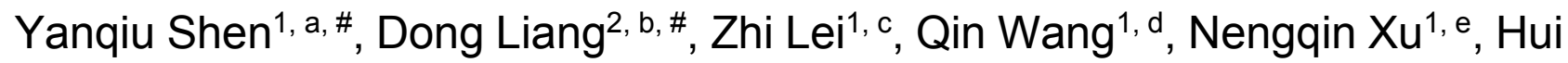 \\ $\mathrm{Xia}^{2, \mathrm{f}^{*}}$
}

1 College of Horticulture, Sichuan Agricultural University, Chengdu, Sichuan, China 2 Institute of Pomology and Olericulture, Sichuan Agricultural University, Chengdu, Sichuan, China a517590097@qq.com, b756141959@qq.com, c1280195400@qq.com, d18428385472@163.com, exunengqin@163.com, fsusanxia_2001@163.com

\#Co-first author, *Corresponding author

Keywords: melatonin, kiwifruit, weak light, phenolics substances, antioxidant activity

Abstract: In this study, kiwifruit seedlings were used as the materials, were applied $100 \mu \mathrm{mol} / \mathrm{L}$ melatonin (MT) solution by root irrigation, to investigated the effects of MT on kiwifruit' antioxidant activity under low light. The results showed the content of TPC, TFC and TFAC of kiwi leaves under MT treatment were significantly higher than that under water and weak light treatments. The antioxidant capacity was measured by three different methods (FRAP, DPPH and ABTS), showed MT treatment significantly increased free radical scavenging ability of kiwi leaves. The indexes of MT treatment showed a trend of first increased and then decreased and last increased. The results provided a theoretical basis for improving the yield and quality of kiwifruit.

\section{Introduction}

Kiwifruit is the vine deciduous plant belonged to Actinidiaceae Actinidia, which also called 'longevity fruit' due to its high content of $\mathrm{VC}{ }^{[1]}$. China is the origin of kiwifruit, with rich wild kiwi resource ${ }^{[2]}$, and also the biggest producer of kiwi. However, kiwifruit production is often susceptible to a variety of stresses, such as low light, drought, and other factor, often resulting reduction of yield. Low light environment abound in main growing area of kiwifruit in China, e.g., the southwestern of Sichuan. MT is an indole small molecules, the chemical name is $\mathrm{N}$-acetyl-5-methoxytryptamine ${ }^{[3]}$. It has a variety of functions on plant, including the regulation of plant growth and development, such as changing the florescence ${ }^{[4]}$, anti-aging ${ }^{[5]}$. All the functions are beneficial to improve the biological production of plants. Therefore, it is of great theoretical and practical significance to study the influence of applying exogenous MT on kiwi antioxidant activity

\section{Materials and methods}

Plant materials and treatments Seeds of kiwifruit were collected in September, 2015. After stored at $4{ }^{\circ} \mathrm{C}$ for two monthes, seeds were treated with variable temperature at $4{ }^{\circ} \mathrm{C} 16 \mathrm{~h}, 24^{\circ} \mathrm{C} 8 \mathrm{~h}$ for two weeks, and then, placed at $25^{\circ} \mathrm{C}$ for 10-15 days to germinate. Sowed the sprouting seeds, and placed them in the artificial climate chamber (light-dark cycle: $12 \mathrm{~h} / 12 \mathrm{~h}$, diurnal temperature: $25^{\circ} \mathrm{C} / 20^{\circ} \mathrm{C}$ ). When had 2-3 true leaves, seedlings were moved into pots. 
We chose uniformly seedlings to treat with MT and weak light: (1) T1: the plants were put under normal light and irrigated with water; (2) T2: the plants were placed under the shade with $60 \%$ sunlight, irrigated with water; (3) T3: seedlings were irrigated with $100 \mu \mathrm{mol} / \mathrm{L} \mathrm{MT}$ solution, $20 \mathrm{ml}$ each pot, once every two days, after five times, transfered to under shade with $60 \%$ sunlight. The day seedlings were transfered to under shade was designed as 0 days. Every treatment used 15 pots, 3 seedlings every pot. Leaves (5-9 leaf position) were sampled on $0,2,4,6,8$ days. All collected tissues were immediately frozen in liquid nitrogen and stored at $-80^{\circ} \mathrm{C}$.

Determination of TPC, TFG, TFAC, FRAP, DPPH and ABTS The total phenol content (TPC), total flavanone content (TFC) and total flavanols content (TFAC) was determined according to the method of Xiaoqian Wang ${ }^{[6]}$, (TPC were expressed as gallic acid equivalent, and TFC were expressed as rutin equivalents and TFAC were expressed as catechins equivalent). The radical scavenging ability of DPPH and ABTS, and ferric ion reducing capacity (FRAP), adopted Guorong $\mathrm{Du}^{[7]}$ ( results were all expressed in TE equivalent value). All indexes held nine repetitions, the average value were used as the measured values.

Data analysis Excel 2010 was used for data processing and graphing. Analysis of variance was performed by the SPSS software (SPSS, Inc., Chicago, IL, USA). Each processing held 9 repetitions. Significant differences were detected by Duncan's test at the $\mathrm{P}<0.05$ level.

\section{Results}

Change of phenolics substances content Phenolics in plants can clear the radicals caused by stresses, so phenolics play an important role in the resistance physiology of plants. During the processing of this test, application of MT significantly improved the content of phenolics substances under weak light treatment (Fig.1 A, B, C). Such as T3, the content of TPC and TFAC had increased steadily, reached the maximum at day 8 . Compared with day 0 , the content of TPC has increased $56.3 \%$ at day 8, was simultaneously 1.5 times of T2 and 1.2 times of T1 (Fig.1 A). And TFAC has also increased by $92.3 \%$, while TFC had increased by $218 \%$ at day 2 , then began to reduce, but at day 8 , still significantly higher than T1 and T2. The contents of TPC, TFC and TFAC in plants showed same tendency that increased firstly and then decreased and increased.
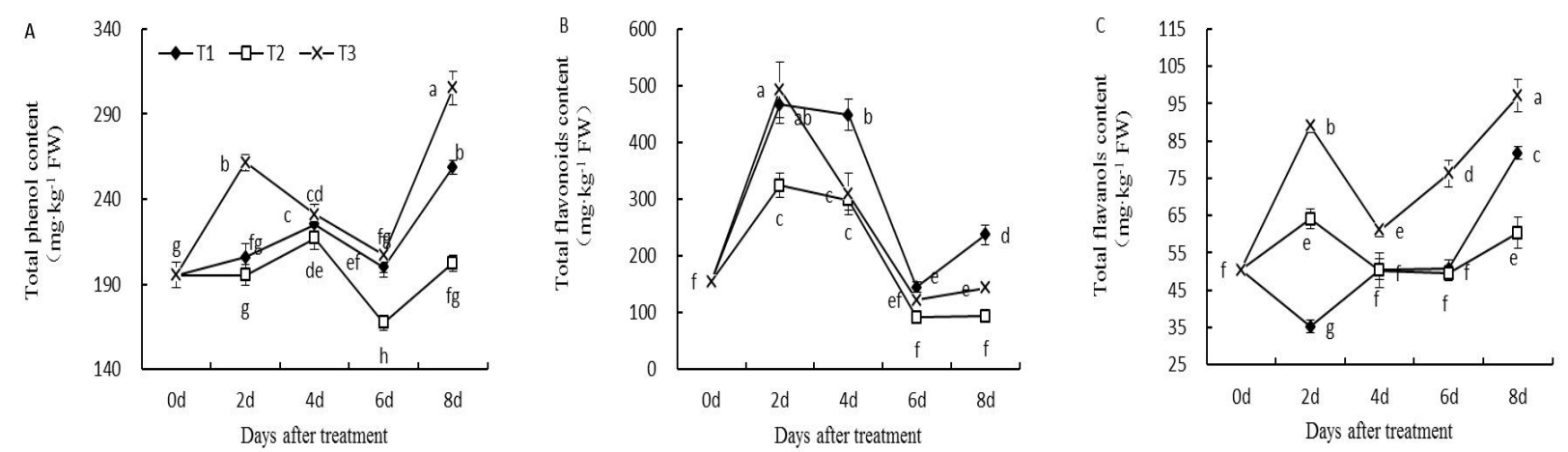

Fig. 1 Effects of MT application on contents of TPC, TFC and TFAC of kiwifruit leaves. Changes of the antioxidant capacity FRAP, DPPH and ABTS were common methods to determinate antioxidant activity. MT application also significantly improved the kiwifruit's antioxidant capacity. The FRAP mainly reduced $\mathrm{Fe}^{3+}$ to $\mathrm{Fe}^{2+}$, the reducing power of T3 were significantly higher than $\mathrm{T} 1$ and $\mathrm{T} 2$ all the time (Fig. 2 A); while the antioxidant capacity of T2 were also basically significantly lower than T1, which indicated weak light can make the reduction ability of kiwi significantly dropped, and had certain influence on the normal growth of kiwi. DPPH showed that leaves' radical scavenging ability was also significantly higher than other 
treatments (Fig. 2 B). ABTS, free radical scavenging capacity of T3 dropped first, and then rose again, but little change as a whole; after 6 days of treatment, T3 had remarkable difference with T2, but in the early stage had little difference (Fig.2C) All of the methods showed a trend of increase.
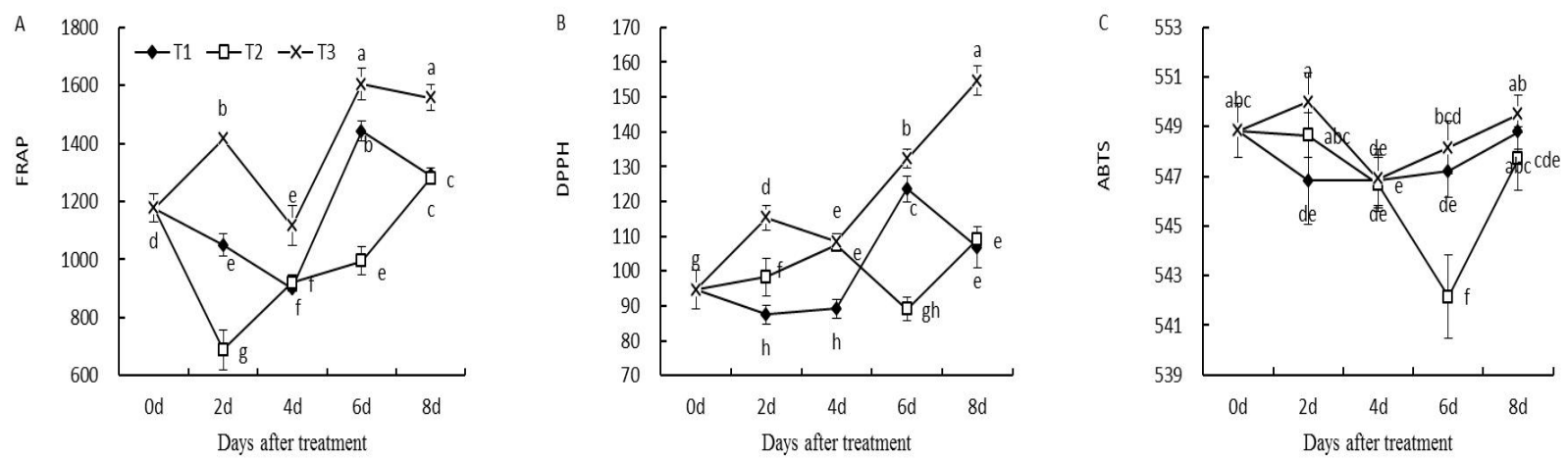

Fig. 2 Effects of MT application on antioxidant capacity (measured by FRAP, DPPH and ABTS).

\section{Discussion}

Plant growth was influenced by light, temperature and other environmental factors. Those bad factors not only affect the growth and development of plants, but also cause plant tissue damage or even aging, excessive membrane damage lead to the accumulation of reactive oxygen species in the plant ${ }^{[8-11]}$. Similarly, weak light conditions can also lead to a large number accumulation of active oxygen in plants, and affected cell metabolism and plant damage.

Phenolics substances are antioxidant substances that scavenge active oxygen in plants. Studies have shown that biotin flavonoids have strong antioxidant ability to clear free radicals in plants due to stress, e.g., the hydroxyl group of the flavonoids can provide electrons, scavenging reactive oxygen species ${ }^{[12,13]}$. In this test, MT treatment significantly increased the content of T3'phenolics. TPC, TFC and TFAC showed the same tendency, maybe the MT activated the activity of enzymes related to the synthesis of phenolic compounds; and firstly increased possibly due to the adaptation to the weak light. But in different indicator, T1 and T2'trends varied, probably different phenolic substances response difference to weak light stress.

DPPH, ABTS and FRAP were three wide used methods for the determination of antioxidant activity, which can comprehensively evaluate the antioxidant capacity of plants ${ }^{[14]}$. ABTS and DPPH were used to determine free radical scavenging ability ${ }^{[15,16]}$. In ABTS method, the ABTS ${ }^{+}$ free-scavenging ability of T1, T2 and T3 were not significant, probably due to the content of peroxides (for example, $\mathrm{H}_{2} \mathrm{O}_{2}$ ) in leaves was not significant, and can't form $\mathrm{ABTS}^{+}$cationic radicals with different amounts of $\mathrm{ABTS}^{+}$and peroxidase. FRAP [17] is an assay determine $\mathrm{Fe}^{3+}$ reduction antioxidant capacity. The comprehensive evaluation results of three methods showed the antioxidant capacity was improved under weak light, which was different from Ling Shao ${ }^{[18]}$, probably the reason that different plants response differently to the same stress. Simultaneously, MT treatment significantly improved the antioxidant capacity, maybe the improvement of antioxidant enzyme activity and antioxidant substances content lead to the comprehensive results.

\section{Conclusion}

In summary, the results of MT treatment showed a tendency that increased firstly, and then, decreased on the whole. After MT treatment, the content of TPC, TFC and TFAC have significantly increased. According to qualitative analysis result of three methods (ABTS, DPPH 
and FRAP), MT application also significantly improved the free radical scavenging ability of kiwifruit. Therefore, for the weak light condition in production, MT application can efficiently solve the problem of accumulation of reactive oxygen species in the plant. Apart from that, MT increased antioxidant substances' content under stress, moreover, improved its antioxidant capacity, so as to enhance its resistance; which provide theoretical basis for the rational use of MT.

\section{Acknowledgements}

This work was financially supported by the Sichuan Technical Supports Foundation (2016NZ0105), Training Programs of Innovation for Undergraduates in Sichuan (201610626057).

\section{References}

[1] X.X. Xue: Fresh fruit processing technology and process formula (Science and Technology Literature Publishing House, Beijing 2005).

[2] J. Ding: Sichuan Agricultural University (2006).

[3] P. Wang: Northwest A\&F University (2015).

[4] M.X. Liu: Northwest University (2015).

[5] N. Zhang, H.J. Zhang, R.C.Yang, et al.: Agricultural Science \& Technology Vol. 13(9) (2012), p. $1833-1837$

[6] X.Q. Wang: Northwest A \& F University (2015).

[7] G.R. Du: Northwest A\&F University (2009).

[8] X.X. Chen, L. Li, H.B. Niu, et al.: Journal of Henan Agricultural Sciences (12) (2008), p.3840.

[9] C.Y. Chen, H.D. Wang and Y. Ding: Acta Horticulturae Sinica Vol. 32(5) (2005), p.911- 913.

[10] Q.M. Wang: Journal of Agro-Environment Science Vol. 25(2006) (4), p. 918- 921.

[11] X.L Hou., J.F. Li, and X.Y. Xu: Acta Horticulturae Sinica Vol. 29(2) (2002), p. 123-127.

[12] C.G Chen and F.X. Han: Journal of Anhui Agricultural Sciences Vol. 34(13) (2006), p. 29492951.

[13] Y. Zhang, G.Q. Zhang, P.F. Zhan, et al: Hebei Journal of Forestry and Orchard Research Vol. 21(3) (2006), p. 311-315

[14] X.Y. Wang, G.R. Du and H. Li: Journal of Food Science and Biotechnology (3) (2012), p. 247-252.

[15] N.J. Miller and C.A. Rice-Evans: Clinical Science, Vol. 84(1993), p. 407-412.

[16] O.I. Aruoma: Mutation Research. Vol. 523-524(2003), p. 9-20.

[17] K.S. Shivashankara, S. Isobe and M.I. Ai-Haq: J Agric Food Chem, Vol. 52(2004), p. 281-1286.

[18] L. Shao: Journal of Zhaoqing University Vol. 31(5) (2010), p. 43-47. 